\title{
Faculty use of the course management system iLearn at the American University of Sharjah
}

\author{
David Prescott \\ with Cindy Gunn, Walid Alieldin, Kathleen Botter, \\ Shireen Baghestani and Hamed Saadat
}

American University of Sharjah

\section{Introduction}

That course management systems (CMS) such as Blackboard and Moodle are relevant to higher education is beyond question. Tertiary institutions invest significant resources in developing CMS and promoting their use by faculty members. Considerable research has been undertaken with respect to cost-effectiveness, to the 'buy as opposed to build' quandary and also to CMS product rating. Far fewer studies have been devoted to examining how course management systems are being used in higher education, whether such use is effective and what views the faculty members who use/do not use the technology have about such systems. The American University of Sharjah (AUS) has, since 2004, made a strong commitment to CMS through investment in iLearn (the AUS version of Blackboard) as a teaching and learning medium. This paper reports on initial findings from an investigation, conducted by a cross-disciplinary, cross-generational research team of faculty, staff and students. The study seeks to gain a clear appreciation of how iLearn is being used at AUS and to recognize the faculty members' opinions and perceptions of the CMS iLearn.

\section{Context}

The study reported on here used Morgan's (2003) study at University of Wisconsin System (UWS) as a starting point. Morgan's study was conducted to investigate how faculty members used the CMS, to identify what features were regularly used and in what learning environments, and to ascertain what circumstances would induce greater usage. We used the Morgan investigation as a foundation and endeavored to build on that work but with clear awareness of the technological innovation that has ensued since the UWS study in 2003. Some similarities remain. The American University of Sharjah (AUS), as with UWS, has a strong commitment to CMS through investment in iLearn as a future teaching and learning medium. The tools available in iLearn can be divided into administrative, organizational and interactive categories; our research endeavored to determine the patterns of usage among faculty members. The research team was gender balanced and crossgenerational. It also drew on faculty, staff and recent graduates from different departments of the university.

The following questions guided the study

- For what purposes is iLearn being used by faculty?

- What factors encourage faculty to increase or decrease their usage?

- What pedagogic gains does iLearn facilitate?

- What examples of helpful practice can be identified by faculty? 
A research community was established on iLearn [org_iLearn_RG] for sharing minutes of meetings, research articles and other relevant information. The effectiveness of this community was proven at the end of the research period when the report was being compiled: the community allowed all team members to access the stored information and accumulated knowledge and contribute efficiently to the report.

\section{Literature review}

The study conducted by Morgan (2003) was extensive, involving a 31 item survey of 730 faculty and institutional staff and semi-structured interviews with 140 UWS faculty and institutional staff, as well as manual counting and examination of CMS usage logs. Participants in the study represented about $11 \%$ of the UWS faculty across two doctoral-research institutions and nine freshman-sophomore campuses. Both Blackboard and WebCT were being used (by $74 \%$ and $22 \%$ of faculty respectively); $43 \%$ of the CMS users (as at November 1, 2002) had experience of using a CMS other than Blackboard and in the Fall 2002 semester the CMS formed part of the instruction in 5,160 courses.

The key findings of the UWS study are the following. The students were not as tech savvy as many faculty believed and in fact the study revealed that students had insufficient skills to use CMS without training. Faculty revealed a number of reservations which led to poor CMS uptake rates. Principal amongst these were fear of loss of control of instruction and fear of the CMS instructional environment and also a belief that the inflexibility of CMS procedures would undermine pedagogy. This reluctance was general, not particular to a specific CMS, as there was no support amongst this population for change to an alternative system. Where faculty did adopt the CMS, the capacity of the system to undertake routine, repetitive organizational tasks was valued above other considerations. The study showed, not surprisingly, that faculty reported limited use of CMS functionality, with strong focus on the 'static' tools (SafeAssign, surveys, quizzes and tests). Faculty adoption of CMS was most significantly encouraged by strong departmental and executive leadership by example. The UWS study also found that training was crucial. The favored model for this training was small-scale, department- or course-based training with academic colleagues, focused on real examples rather than on generic models. A number of UWS faculty expressed the view that faculty members learn as much from their peers as they do from CMS trainers. There was no reported evidence that showed that the CMS improved pedagogy.

Hamuy and Galaz's (2010) study at the University of Chile, which assessed the first two years of implementation of Moodle, indicated a preference amongst faculty and students for informational interaction and resource use as opposed to using Moodle for communication purposes. The authors consider the study findings inconclusive on the question of whether more learning or better learning is taking place. To answer that question a longer, more profound qualitative study is recommended. Blin and Munro (2008) studied the virtual learning environment (VLE) at Dublin City University and concluded that despite widespread usage of the VLE little change in the structure of teaching and learning had occurred. The VLE was mainly used for administrative and dissemination purposes and complemented or replicated existing practices. Furthermore static, content-based resources such as web pages and lecture notes tended to be the main learning materials added to the VLE. Blin and Munro attributed this situation to lecturers' lack of competencies and a training and development system which had not addressed the actual needs of the lecturers. They pointed out that training needed to address not only the issue of effective use of the VLE tools but also how to structure tasks that achieve some learning purpose. In this respect they tended to agree with the UWS study, which also highlighted appropriate training as a clear need.

In another study that reflected some of the misgivings reported from faculty in the UWS investigation, Lane (2009) contended that technologies are not pedagogically neutral but that "they influence pedagogy by presenting default formats designed to guide the instructor toward creating 
the course in a certain way" ( $p$ 2). Lane claimed that this is true of Blackboard/WebCT and also of newer, more constructivist oriented systems such as Moodle. Most studies focus on ease of use or on how faculty use CMS; very few discuss the way these systems influence pedagogy. Added to this issue is that many new online teachers are Web novices - required to use the systems but with a lack of in-depth understanding about effective use of online technology. This observation reflects one of the contentious claims in an earlier article (Lane, 2008), where the assertion that "more and more people are becoming CMS users because they feel pressured to, not because they want to" is made (p. 5). The implication is that people in this situation would be likely to use the obvious management tools rather than uncover pedagogic possibilities.

Lane's (2008) evidence is observational and incidental. It is based on conference attendance engagements, blogging, interactions with faculty and surveys of instructors at two community colleges. It is not a very systematic collection of data; rather more literature review and impressionistic data retrieval. The main discussion in Lane's article centers on the following issues. Enterprise-scale systems were created to manage traditional tasks (grade posting, test creation, enrollment management) and the built-in pedagogy is based on traditional presentation and assessment approaches. There is a channeling of activity into lectures, discussions and exams, "reinforcing uncritical acceptance of the traditional features of the classroom model" ( $p 3)$.

Other studies that have relevance to the AUS investigation include work conducted by Sheely (2008) and related work by $\mathrm{Li}$ and Ranieri (2010). Sheely explored the misconception that students are "digital natives" because they have grown up with computers, software applications, internet access, and related communication tools. The focus of Sheely's paper was that we need to be aware of the following learning principles as they apply to our own students: students construct knowledge, they learn through interaction, they are social (learning in communities), they learn through authentic experience and they seek relevance. Students learn this way not because they are Digital Natives but because they are human beings. This is how humans learn, by constructing knowledge through authentic experiences in social situations. Technology is just one of many tools we can use to achieve this. It is more beneficial, Sheely concludes, to design learning environments which will engage learners to construct knowledge, to learn in communities and to seek relevance. The relevance to the current study is that the combination of students and computers does not necessarily lead to effective learning. Li and Ranieri's work involved the use of an Instant Digital Competence Assessment Tool developed at the University of Florence. This study conducted with Chinese teenagers concluded that the role of education is a more influential factor than constant exposure to computer hardware. Both studies noted the importance of well-designed teaching and learning materials, a factor also alluded to by Blin and Munro (2008).

Selwyn's (2007) work explores how university faculty use of computer technology is marginalized and its use curtailed in a number of ways. Selwyn identifies a consistent theme from evidence in national policies through to how students use computer technology in tertiary institutions: this use he says is constructed in limited, linear and rigid terms. This is rather different from the creative, productive, and empowering uses celebrated by educational technologists. In the light of these constraints, the paper considers how these dominant constructions of a peripheral and limited use of ICT may be challenged. In particular, Selwyn recommends critical thinking about how to foster a more expansive and empowered use of computer technology within university settings from within universities and departments of universities rather than the broad, policy-driven, generic models that have failed to instigate innovative change.

Finally McPherson \& Nunes (2008) reported on critical issues for e-learning delivery and identified critical success factors related to e-learning strategies, implementation, design and delivery. The data for their study was collected through focus group interviews with approximately 15-20 participants in each of four separate workshops at various e-learning and educational technology conferences: AUA 2002, E-Learn 2002, ICALT 2002 and ICCE 2002. Respondents clearly recognized 
the necessity for systematic identification of training needs through programs of staff review and development. This relates to the UWS finding concerning training. Furthermore, respondents observed that if top-down strategies in universities are devised to implement e-learning strategies, then appropriate levels of staffing and support should be put in place. Without these, the sustainability of e-learning cannot be guaranteed.

Clear themes permeate these studies: the need for appropriate technical training and development in localized settings, as well as the need for appropriate tasks and activities. The second theme also requires appropriate training so that teachers are able to utilize the interactive applications embedded in CMS applications in ways that are pedagogically effective and which pay attention to developing students' higher order cognitive skills.

\section{Methods}

Three methods of data collection were used in this study; survey, interviews and focus groups. The AUS Institutional Review Board approved the three investigative methods in April, 2011 with written confirmation following on May 11, 2011.

\section{Survey}

A primary reason for this investigation was that relatively few studies have been devoted to the views of those (faculty and staff) expected to use Course Management Systems (CMS) like iLearn. The research team in this study believed that these opinions and perceptions are important and need to be heard.

The survey focused on two areas of concern: the iLearn tools used (e.g. Announcements, Wikis) and the purposes for which these tools were used (e.g. Cater to diverse learning styles, Supplement lecture material). In each case a four point scale allowed the respondents to indicate their own level of use from consistently through occasionally to almost never and never. No neutral option was provided in order to preclude default selections of a non-indicative response. Two further sections asked for indications and comments on features and situations that would encourage greater use of iLearn and those that discouraged use. A final section offered faculty who wished to be interviewed to indicate this. The survey was distributed through iLearn to all 394 AUS faculty and utilized the anonymity functions offered by the CMS software with a link access so no login was required. The survey was opened on two occasions: 24 April-8 May, 2011 and 31 May-2 June, 2011. 98 responses were received, a return rate of 24 per cent. This compares favorably with the UWS study which surveyed approximately 11 per cent of faculty. The 98 responses were from all schools in the university in the following pattern:

- 48 per cent College of Arts \& Sciences

- 22 per cent College of Engineering

- 13 per cent College of Architecture, Art and Design

- 12.5 per cent School of Business \& Management

- 02.25 per cent Library

- 02.25 per cent Academic Achievement Academy

The faculty numbers in the Colleges of AUS vary and the responses reflect this variation fairly well. At the time of the survey the College of Arts \& Sciences included $45 \%$ of the total AUS faculty, College of Engineering 23\%, College of Architecture, Art \& Design 14\% and School of Business \& Management $18 \%$. 


\section{Interviews}

Sixteen faculty and/or staff agreed to be interviewed, representing an 18 per cent response rate. Interviewees represented all colleges and schools in the university as well as the Library. The interviews were conducted by the research assistants, Ms. Shireen Baghestani and Mr. Hamed Saadat. Training to standardize the interview process and to inculcate effective interview technique was provided by Faculty member, Mrs Patricia Prescott, Department of Writing Studies. The purpose of the training was to ensure, as far as possible, uniformity in the way in which the interviews were conducted, to ensure the same questions in the same order were put regardless of interviewer. Use of the TEDS [tell/explain/describe/suggest] approach allowed the interviewers to probe where they felt extra information might be forthcoming but to do so in manner that is nonthreatening. Both the training and the use of the TEDS approach relate to comments about consistency and reliability made by other researchers. Patton (2001), for example, believes that it is usually necessary for researchers to develop an interview schedule which lists the wording and sequencing of questions to ensure consistency. Lindlof and Taylor (2002) point out that interview schedules are often considered a means by which researchers can increase the reliability and credibility of research data.

Interviews were used in this study to seek confirmation of findings that emerged from the survey data, to allow for clarification/expansion on issues of interest to the interviewees and to allow for any new information to emerge. While attempting to draw out more particular information, the context of personal structured interviews nevertheless follows a patterned procedure in order to minimize the impact of context effects. Interviews were conducted in faculty offices and at times nominated by interviewees; permission to record interviews was sought verbally prior to commencement. Interview findings based on transcription of the exchanges were sent to each participant for confirmation before analysis; a number of minor inaccuracies were rectified through this process.

Interviews with sixteen faculty were conducted in the week following the initial opening of the survey, that is, May 9 through to May 16, 2011. Interviews were recorded using non-intrusive 4GB Sony Voice recorders which have the advantage of microphone sensitivity and compact size.

The sixteen files of interview data were transferred into text files using a combination of Dragon Naturally Speaking Premium 11 Voice recognition software and manual transcription. In general we found the use of the voice recognition software (VRS) to be only partly reliable. The combination of automatic and manual transcription of the interview data was a sensible compromise.

The interview data was presented for analysis during Spring Semester, 2012. Nvivo 9, a qualitative data analysis (QDA) computer software package, was partially used for this purpose. However we found the software rather cumbersome to use and experienced some difficulty fitting our interview data into the Nvivo frameworks. We therefore used some Nvivo analysis but more frequently we worked in small analysis teams of two or three, identifying and comparing themes and confirming those where agreement was reached as the result of independent analysis.

\section{Focus Groups}

We convened three focus groups, one each from the College of Arts and Sciences, the College of Engineering and the College of Art, Architecture and Design. Three faculty members, one from each of the Colleges previously mentioned volunteered to convene focus groups and each asked for volunteers from his/her respective College. With only one exception the participants in the focus groups had not been involved in the interviews.

Focus group discussion can often produce insights stimulated by the interaction of a group setting. Lindlof \& Taylor (2002, p. 182) describe this group effect as "a kind of 'chaining' or 'cascading' effect; talk links to, or tumbles out of, the topics and expressions preceding it." According to Gamson 
(1992) and Morgan \& Krueger (1993) focus groups allow a large amount of interaction on a topic in a limited period of time. They encourage a greater variety of interactions between participants than survey or interview and they encourage more open discussion. One limitation is that the more the researcher attempts to direct or focus the group, the less naturalistic the setting becomes and therefore the less naturalistic the data. If the researcher withdraws then a further limitation that may occur is loss of focus and the emergence of data which may not address the issues at hand.

Focus groups have an advantage over individual interviews in that they allow interaction and encourage similarities and differences regarding opinions and experiences to emerge as opposed to reaching such conclusions by post hoc analysis of separate statements from each participant. However interviews are more easily guided by the interviewer and more time is generally available for each individual than in a focus group, which also generally provides less depth and detail about opinions and experiences of any one participant.

For the focus groups in this study there was control only in the guiding questions: we removed researchers from the focus groups entirely. This means that we can accept that the data that emerged as based on the issues that concern faculty and that the "insights springing from the homogeneous, social situations" (Agar \& MacDonald, 1995, p. 84) are those perceptions which are the concerns of the participants. The conveners recorded the discussions; the recordings were subsequently transcribed and the transcriptions returned to the appropriate groups for confirmation prior to analysis.

\section{Findings}

\section{Survey}

Figure 1 shows the results relating to the survey questions about which iLearn tools respondents used. It is clear from this array that the 'static' tools (Grade Center, Email, Announcements and Posting Content) are used more extensively than the interactive tools such as wikis, blogs and discussions. In this regard the findings reflect those of Morgan's (2003) study which reported strong focus on the 'static' tools. Similarly the studies of Hamuy and Galaz (2010), Blin and Munro (2008) and Selwyn (2007) all revealed that faculty use patterns favoring the administrative management aspects of technologies as opposed to the more pedagogically interactive possibilities which exist both in both CMS in particular as well as in modern computer technology generally.

Morgan's (2003) study found that faculty favored the capacity of a CMS to take on organizational tasks (posting grades, posting announcements) in preference to engagement with aspects of the system that might be integrated into their teaching methodology, such as the use of blogs and discussion boards, for instance. Clearly the survey results shown in Figure 1 cannot be interpreted in this way without confirmatory evidence; however it is possible that the apparent reluctance to engage with the interactive aspects of iLearn indicated in Figure 1 might result from factors that emerged from the UWS study and which have also been found significant in other studies. One of these factors is training, and in this regard AUS provides reasonably extensive faculty development assisting new appointees to engage productively with iLearn. For instance, iLearn Training For Faculty is a three module course which aims to provide faculty with online training in three key areas: Building Courses, Enhancing Communication and Assessing Learners. Faculty complete the modules they choose in their own time, with online support available asynchronously. Similarly, a broad introduction to iLearn is part of orientation for all new faculty members at AUS and ongoing support is always available through the iLearn Helpdesk. Academic Computing officers can be available for one-on-one consultations in faculty members' offices if required. So in these terms AUS appears to be providing strong institutional training support. 


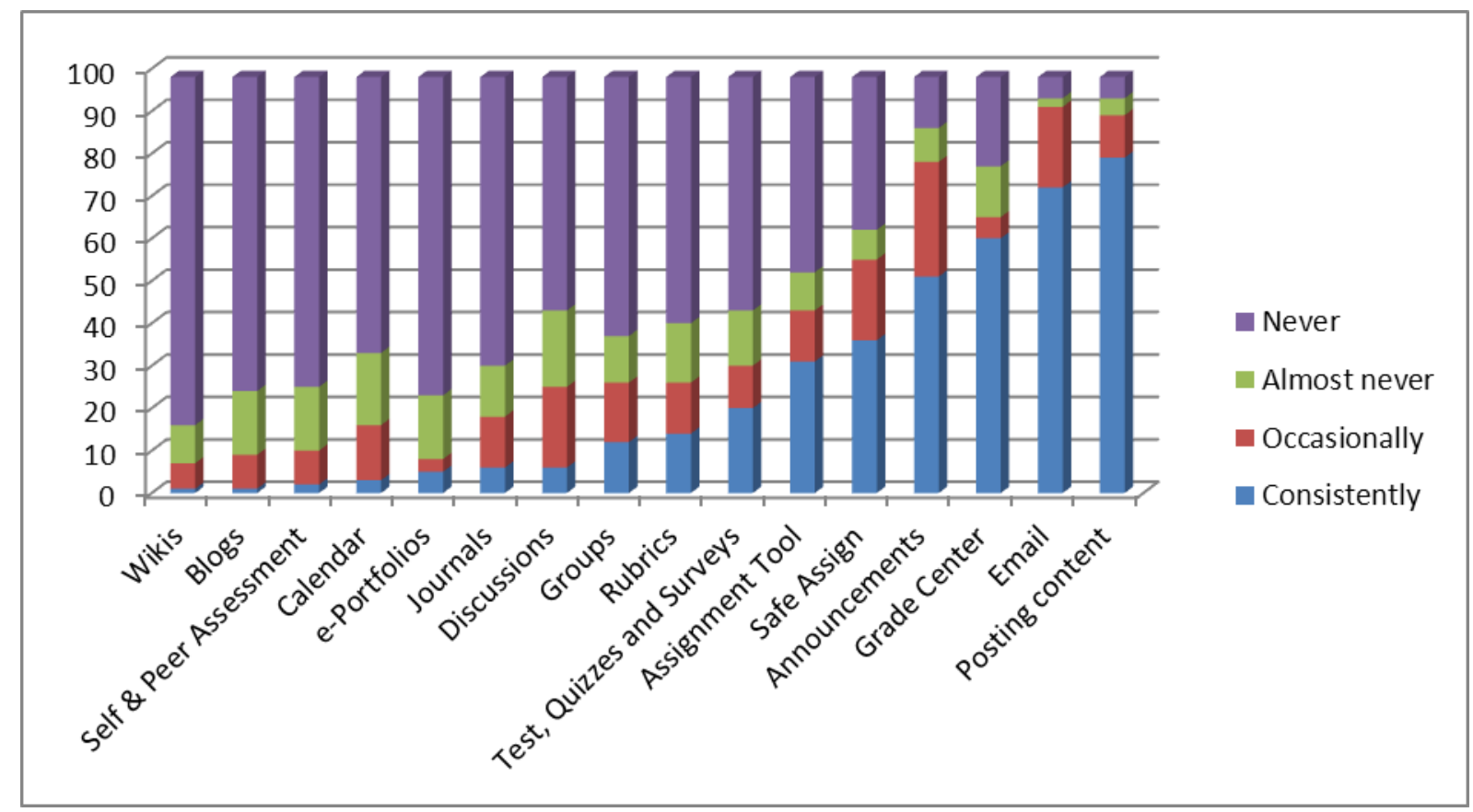

\section{Figure 1: CMS tools favored by AUS survey respondents.}

However, what is clear from Morgan's study and other research on IT uptake by teachers (Chambers $\&$ Bax, 2006) is that training which is small-scale, department- or course-based with one's academic colleagues is more popular than generic training. At UWS this preference was shown to be because of the focus on relevance that small-scale training with peers engenders. Such training takes place in a situation that is germane to all participants and has a high degree of immediate application. The AUS training described above does not fit this model.

Figure 2 shows the purposes for which the iLearn tools are used. Again it is clear that the organizational tools of iLearn are favored by the respondents as the interactive, pedagogically flexible tools are again shown to be less strongly supported. While the results tend to confirm the preferences indicated by respondents with respect to tools used there is less obvious separation between static and interactive tools in this focus on purpose. The results suggest that faculty may in some instances view functionality independently from the tools that are supposed to promote such pedagogy. This would not be surprising since it would tend to agree with findings in the UWS study, where faculty valued integrity of their instructional environments and showed antipathy towards the "inflexibility of the structure of the CMS" (Morgan, 2003, p. 45).

The participants in this study indicated that they mainly use the AUS CMS for administrative purposes such as posting content, sending announcements and posting grades. These findings from the survey are supported by the usage pattern statistics for the Fall semester 2011, which reveal that of 832 active course sites all posted content, 481 (57\%) posted announcements while 243 (29\%) used assignment manager. In the survey improve communication with students was the most strongly supported purpose for using iLearn with nearly $60 \%$ of the respondents indicating that they did this consistently. Other strongly supported purposes (over $40 \%$ consistent use) were supplementing lecture material, increasing transparency and communicating learning expectations. However, as noted above, this communication was one-way in nature. If a commonplace analysis strategy (Macpherson, 1998) is applied to these findings they appear even less supportive of CMS use. MacPherson's strategy involves grouping responses into three categories: 'supported', 'ambivalent' or 'unsupported'. Where 70 per cent or more of respondents strongly agree or agree with an item it is deemed to have been 'supported'; where fewer than 70 per cent but 30 per cent or more of respondents strongly agree or agree with an item it is deemed to be 'ambivalent', and 
where fewer than 30 per cent of respondents agreed or strongly agreed with an item it is deemed to be 'unsupported'. According to this interpretation, the results evidenced in Figure 1 and Figure 2 do not show strong significance.

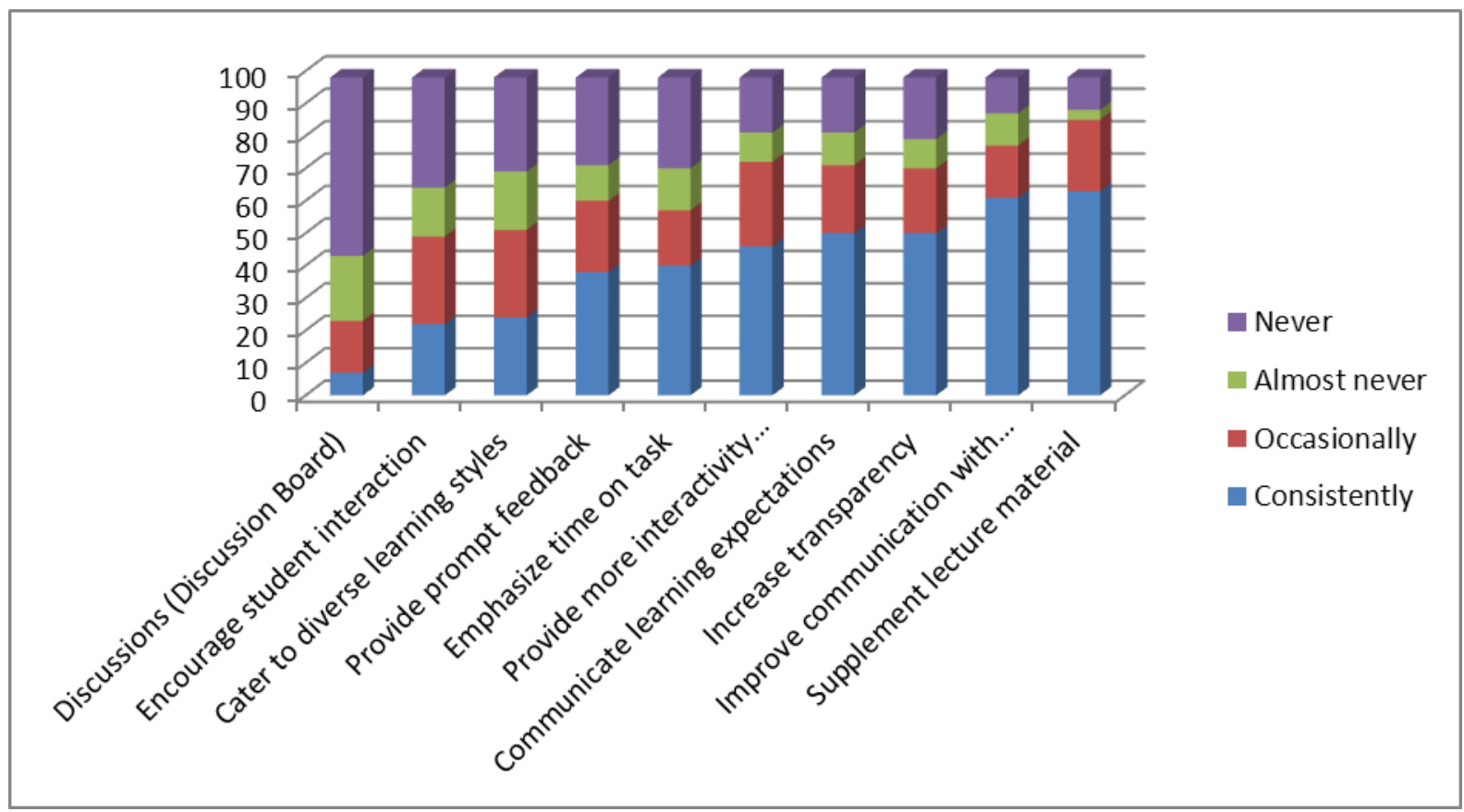

Figure 2: Faculty purposes for use of iLearn.

\section{Interviews}

The interview data was grouped into recurrent themes based on verbal cues: Communication, Course Administration, Ease of Use, Student Expectations, Pedagogy, Training and Tools. Considering the interviews as elements of a case, within-case analysis (Patton, 2001; Moje \& Wade, 1997) was used to search the interview data for confirmation of identified patterns and to discover any additional patterns. This procedure revealed that the interview findings supported the preference for using iLearn for administrative purposes. Consistent opinions expressed in the interviews concerning the positive aspects of ilearn included: "it saves paper", "it saves time", "it is a good way to communicate with students", "it keeps classes organized" and "it provides a secure testing environment through the function lockdown browser".

Furthermore if the interview data is viewed in terms of the balance of positive and negative views within each identified thematic category, an endorsement of the survey findings emerges very clearly. In the category Communication positive views are four times more prevalent than negative views, a pattern repeated in the category Course Administration, where the positive views outweigh the negative by nearly three to one. These results confirm of the survey findings; faculty place value on the management aspect of iLearn.

In the category Ease of Use a sharp reversal of perception is clear, as more than $90 \%$ of the views about iLearn are negative. The situation is less dramatic with respect to the category Pedagogy although negative views are more than twice as frequent as positive views. In the final three categories the results are more mixed. Concerns about the forbidding nature of the iLearn interface vis-à-vis Student Expectations, and the need for Training to be department focused are clear in the data. The perceptions that emerge with respect to the different Tools of iLearn are ambivalent, with support and criticism for discussion board, surveys, grade book in more or less equal measure. 


\section{Focus Groups}

Analysis of the transcripts of the three focus groups revealed observations and opinions strongly related to administrative and communication functions and purposes. This was particularly notable in responses to discussion about benefits to students and faculty. Typical responses included: "24/7 mobile access, easy communication", "test and homework solutions can be posted" and "it streamlines student/faculty interaction". In this respect the focus groups reflected the survey and interview findings, with emphasis on the static tools of iLearn. In one of the focus groups the convener summed up the exchange on this issue, "so communication seems to be the big thing". Further benefits that rated smaller mention included ease of use; there was some mention of the use of the survey tool, of shy students being encouraged to participate by the anonymity of the online environment and the use of the anonymous peer review.

Focus group responses to the downsides of using iLearn revealed a number of observations concerning pedagogy. In this regard the responses reinforced the trend that emerged from the interviews and commented on earlier. A recurrent theme, which emerged also from the interviews, was the disenfranchising, reductionist nature of the CMS. One focus group stated it this way:

- 'it makes the faculty less aware of the students' interests, there is less face-to-face communication

- you do not build a substantial relationship with your students

- students do not improve their oral communication and presentation skills

- you may not know if the student has understood or not because you can't observe the facial expression of the student"

We viewed the focus group findings in this study as a contrast between our expectations and realities. We expected to probe for insights emerging from the manner focus group "participants both query each other and explain themselves to each other" (Morgan \& Krueger, 1993, p. 139). However, unfortunately in this case focus group conveners' efforts to guide discussion may well have had the effect of curtailing interaction and producing a consensus effect (cf. Agar \& MacDonald, 1995).

\section{Discussion}

In answer to our first research question, "For what purposes is iLearn being used by faculty?", it is clear that administration, management and pragmatic communication are the most common purposes. The survey results are supported by the interview and focus group findings and reinforced by the usage patterns for Fall semester 2011 reported earlier in the paper. Using a CMS in these administrative management and communicative ways has advantages for students and faculty: typical affirmative observations include "announcements can be sent out to students easily"; "keeps history of grade changes; provides a permanent backup of data" and "good for dealing with large numbers (students and test items)".

Considering the evidence relating to the second research question, "What factors encourage faculty to increase or decrease their usage?", the indications are that 'ease of use' factors tend to discourage uptake and usage. There are various pedagogic features buried in most current versions of CMS; but finding them easily seems to be the problem. The survey data, the interview responses and the focus group evidence concerning usage patterns of iLearn tools such as discussion boards, blogs, wikis and journals revealed that few faculty members at AUS are using the tools which could encourage students' interactivity. The surveys showed for instance that wikis, blogs, journals and eportfolios are consistently used by less than $5 \%$ of faculty with the never responses well in excess of $70 \%$ for each of these tools. Again the usage patterns statistics for the Fall semester 2011 support 
this data, with the discussion board tool (the most used) recording $7.9 \%$ usage while the wiki (3.7\%), blog and journal tools (2\%) recorded insignificant use.

The interview and focus group findings generally confirm these figures. In the interview data there are some positive observations about the interactive tools: "iLearn lets me be a lot more flexible and incorporate current events better into my class"; "it links to media resources and images" and "discussion board is a wonderful tool I think". However, there are many more negative comments about the pedagogic difficulties involved in using iLearn: "not oriented to teaching"; "it's a dissemination platform rather than an interactive platform" and "it exacerbates spoon-feeding of students". In the focus group data, apart from brief mention of the use of the survey tool, the issue of shy students being encouraged to participate by the anonymity of the online environment and the use of the anonymous peer review function there is little evidence that the interactive tools are used in any significant way.

One clear issue of concern with respect to uptake is the existence of other freely available applications offering greater ease of use and more flexibility. The focus group convened in the College of Art, Architecture and Design captured this issue in the following observation: "for architectural assignments, the drawings get pretty large so they have to use Dropbox instead of iLearn because it doesn't have the capacity. It's a little redundant ...". Another pertinent comment from the same focus group explained the perceptions of iLearn's lack of ease of use ("clunky", "slow", "counter-intuitive") in this way:

\begin{abstract}
for assignments or announcements, you can't upload more than one thing at a time. So for us, if you're uploading some images or something, or lectures which l've done before, you have to click one and upload it, go back, choose another file, upload it. You should be able to do like, like on Flickr, for example, I have a 100 pictures I wanna upload...
\end{abstract}

In terms of the third and fourth research questions, "What pedagogic gains does iLearn facilitate?" and "What examples of helpful practice can be identified by faculty?" the investigation can report very little of substance or value. One reason for this may be that in a system like iLearn the options are not intuitive and need to be explored and learned. Faculty who use these systems are generally not "Web Heads" and most entered online teaching because of a top-down directive rather than because of interest or aptitude. The usual pattern is to adapt offline approaches and techniques to the online environment. Lane (2009) quotes Jones \& Johnson-Yale (2005) who surveyed 2300+ college instructors and showed that the respondents were using email, some discussion forums and plagiarism-check applications; none were communicating with students via video or audio chat or using blogs. Chang's (2008) study, also referred to by Lane, confirms these findings. These findings reflect the Morgan's (2003) conclusion that faculty reported limited use of CMS functionality but a strong focus on the 'static' tools. The same pattern may be said to apply to the current study.

\title{
Conclusion
}

The findings that have emerged in this study have tended to confirm a number of the findings in previous studies elsewhere (Morgan, 2003; Blin \& Munro, 2008; Hamuy \& Galaz, 2010). This is not unexpected because there are commonalities in the contexts. For instance there is a common topdown model of CMS adoption where the organization takes on a large-scale system which is supposed to serve the needs of faculty and students across a significant range of the academic programs. This sort of adoption has the appearance of a 'one size fits all' solution and poses questions as to whether priority is being given to institutional or academic needs in such an adoption.

It is clear from the evidence in this study that there is a strong emphasis on the functional and organizational features of iLearn and far less engagement with the interactive tools such as blogs, wikis and discussion boards. In many ways this imbalance is not surprising. Apart from the factors 
identified by faculty as barriers to uptake, AUS places considerable emphasis on the use of iLearn for administrative management purposes. New faculty's initial experience with iLearn usually consists of a training session on how to use the static tools and the administrative aspects of the CMS. Indeed a respondent in the interviews captured this institutional emphasis succinctly: "It's not a learning management system" (interview informant, May 2011). Where faculty commented on the issue of training, particularly in the interviews there was a clear preference for a change in the way this was delivered at AUS. A strong belief was that "training ought to be more department-focused" and that time was a crucial factor: "faculty need time to learn how to use it properly; a lot of functions are not used to their full potential". It is certainly the case at AUS that after the initial generic training on using iLearn during orientation new faculty members usually find themselves immersed in survival usage and may never move beyond this.

Our results also relate to observations made by Wingard (2004) and Palmer \& Holt (2009) that pragmatic factors tend to be the influences that characterize initial engagement with online work or work through a CMS. However a number of writers have noted that while faculty tend to adopt a CMS as a "mechanism for efficient and accessible delivery of teaching and learning materials to students" (Palmer \& Holt, 2009, p. 379) improved satisfaction and increased familiarity may foster a growth in the range of uses and applications (Morgan, 2003). The evidence of this investigation suggests that this growth has yet to occur in any significant way at AUS.

\section{References}

Agar, M.H. \& MacDonald, J. (1995). Focus groups and ethnography. Human Organization, 54, 78-86.

Blin, F. \& Munro, M. (2008). Why hasn't technology disrupted academics' teaching practices? Understanding resistance to change through the lens of activity theory. Computers \& Education 50(2), 475-490.

Chambers, A. \& Bax, S. (2006). Making CALL work: towards normalization. System 34, 465-479.

Chang, C. L. (2008). Faculty perceptions and utilization of a learning management system in higher education. (Unpublished doctoral dissertation). Retrieved 24 October, 2011 from http://www.ohiolink.edu/etd/view.cgi?acc num=ohiou1210864179

Gamson, W.A. (1992). The social psychology of collective action. In Morris, A. D. \& Mueller, C. M. (Eds.). Frontiers in social movement theory. New Haven, CT: Yale University Press, 53-76.

Hamuy, E. \& Galaz, M. (2010). Information versus communication in course management system participation. Computers \& Education 54(1), 169-177.

Jones, S. \& Johnson-Yale, C. (2005). Professors online: the Internet's impact on college faculty. First Monday, 10(9). Retrieved 16 February, 2011 from http://firstmonday.org/htbin/cgiwrap/bin/ojs/index.php/fm/article/view/1275/1195

Lane, L. M. (2008). Toolbox or trap? Course management systems and pedagogy. EDUCAUSE Quarterly, 31(2), 4-6. Retrieved 23 April, 2011 from http://net.educause.edu/ir/library/pdf/EQM0820.pdf

Lane, L.M. (2009). Insidious pedagogy: how course management systems affect teaching. First Monday, 14(10). Retrieved 23 April, 2011 from http://firstmonday.org/htbin/cgiwrap/bin/ojs/index.php/fm/article/viewArticle/2530/2303

Li, Y. \& Ranieri, M. (2010). Are digital natives really digitally competent? A study on Chinese teenagers. British Journal of Educational Technology, 41(6), 1029-1042.

Lindlof, T. R., \& Taylor, B. C. (2002). Qualitative communication research methods, 2nd edition. Thousand Oaks, CA: Sage Publications. 
Macpherson, R. J. S. (1998). Contractual or responsive accountability? Neo-centralist 'selfmanagement' or systemic subsidiarity? Tasmanian parents' and other stakeholders' policy preferences. Australian Journal of Education 42(1), 66-89.

McPherson, M. and Nunes, J. (2008), Critical issues for e-learning delivery: what may seem obvious is not always put into practice. Journal of Computer Assisted Learning, 24, 433-445.

McQuiggan, C. A. (2006). A survey of university faculty innovation concerns and perceptions that influence the adoption and diffusion of a course management system. Presentation given at the Academy of Human Resource Development International Conference (AHRD), Columbus, $\mathrm{OH}$. Retrieved 4 February, 2012 from http://www.eric.ed.gov/PDFS/ED492812.pdf

Moje, E. B. \& Wade, S. E. (1997). What case discussions reveal about teacher thinking. In Bennett, N. (Ed.). Teaching and Teacher Education, 13(7), 691-712. Exeter: Pergamon.

Morgan, D. L. \& Krueger, R. A. (1993). When to use focus groups and why. In Morgan, D. L. Successful Focus Groups: Advancing the State of the Art. Thousand Oaks, CA: Sage Publications.

Morgan, G. (2003). Faculty use of course management systems. Educause Center for Applied Research. Retrieved 13 October, 2011 from http://www.educause.edu/ir/library/pdf/ecar so/ers/ers0302/ekf0302.pdf

Palmer, S, \& Holt, D. (2009). Staff and student perceptions of an online learning environment: Difference and development. Australasian Journal of Educational Technology, 25(3), 366-381.

Patton, M. Q. (2001). Qualitative research and evaluation methods ( $3^{\text {rd }}$ edition). Thousand Oaks, CA: Sage Publications.

Selwyn, N. (2007). The use of computer technology in university teaching and learning: a critical perspective. Journal of Computer Assisted Learning, 23(2), 83-94.

Sheely, S. (2008). Latour meets the digital natives: What do we really know. In Hello! Where are you in the landscape of educational technology? Proceedings ascilite Melbourne 2008. Retrieved 14 September, 2011 from http://www.ascilite.org.au/conferences/melbourne08/procs/sheely.pdf

Wingard, R. G. (2004). Classroom teaching changes in web-enhanced courses: A multi-institutional study. EDUCAUSE Quarterly, 27(1), 26-35. 